\title{
High-rigidity Forbush decreases: due to CMEs or shocks? ${ }^{\star}$
}

\author{
K. P. Arunbabu ${ }^{1}$, H. M. Antia ${ }^{2,3}$, S. R. Dugad ${ }^{2,3}$, S. K. Gupta ${ }^{2,3}$, Y. Hayashi ${ }^{4}$, S. Kawakami ${ }^{4}$, P. K. Mohanty ${ }^{2,3}$, \\ T. Nonaka ${ }^{4}$, A. Oshima ${ }^{5}$, and P. Subramanian ${ }^{1}$
}

(The GRAPES-3 Collaboration)

\author{
1 Indian Institute of Science Education and Research, Sai Trinity Building, Pashan, 411021 Pune, India \\ 2 Tata Institute of Fundamental Research, Homi Bhabha Road, 400005 Mumbai, India \\ 3 The GRAPES-3 Experiment, Cosmic Ray Laboratory, Raj Bhavan, 643001 Ooty, India \\ ${ }^{4}$ Graduate School of Science, Osaka City University, 558-8585 Osaka, Japan \\ 5 National Astronomical Observatory of Japan, CfCA, 181-8588 Tokyo, Japan
}

Received 2 December 2012 / Accepted 17 April 2013

\section{ABSTRACT}

\begin{abstract}
Aims. We seek to identify the primary agents causing Forbush decreases (FDs) in high-rigidity cosmic rays observed from the Earth. In particular, we ask if these FDs are caused mainly by coronal mass ejections (CMEs) from the Sun that are directed towards the Earth, or by their associated shocks.

Methods. We used the muon data at cutoff rigidities ranging from 14 to $24 \mathrm{GV}$ from the GRAPES-3 tracking muon telescope to identify FD events. We selected those FD events that have a reasonably clean profile, and can be reasonably well associated with an Earth-directed CME and its associated shock. We employed two models: one that considers the CME as the sole cause of the FD (the CME-only model) and one that considers the shock as the only agent causing the FD (the shock-only model). We used an extensive set of observationally determined parameters for both models. The only free parameter in these models is the level of MHD turbulence in the sheath region, which mediates cosmic ray diffusion (into the CME for the CME-only model, and across the shock sheath for the shock-only model).

Results. We find that good fits to the GRAPES-3 multi-rigidity data using the CME-only model require turbulence levels in the CME sheath region that are only slightly higher than those estimated for the quiescent solar wind. On the other hand, reasonable model fits with the shock-only model require turbulence levels in the sheath region that are an order of magnitude higher than those in the quiet solar wind.

Conclusions. This observation naturally leads to the conclusion that the Earth-directed CMEs are the primary contributors to FDs observed in high-rigidity cosmic rays.
\end{abstract}

Key words. Sun: coronal mass ejections (CMEs) - cosmic rays

\section{Introduction}

Forbush decreases (FDs) are short-term decreases in the intensity of the Galactic cosmic rays observed from the Earth. They are typically caused by the effects of interplanetary counterparts of coronal mass ejections (CMEs) from the Sun, and also the corotating interaction regions (CIRs) between the fast and slow solar wind streams from the Sun. We concentrate on CME driven FDs in this paper. The near-Earth manifestations of CMEs from the Sun typically have two major components: the interplanetary counterpart of the CME, commonly called an ICME, and the shock which is driven ahead of it. ICMEs which possess certain well-defined criteria such as reductions in plasma temperature and smooth rotations of the magnetic field (e.g., Burlaga et al. 1981; Bothmer \& Schwenn 1998) are called magnetic clouds, while others are often classified as ejecta. The relative contributions of shocks and ICMEs in causing FDs is a matter of debate. For instance, Zhang \& Burlaga (1988), Lockwood et al. (1991), and Reames et al. (2009) argue against the contribution of magnetic clouds to FDs. On the other hand, other studies (e.g., Badruddin et al. 1986; Sanderson et al. 1990; Kuwabara et al. 2009) have concluded that magnetic clouds can make an

* Appendix $\mathrm{A}$ is available in electronic form at http: //www . aanda.org important contribution to FDs. There have been recent conclusive associations of FDs with Earth-directed CMEs (Blanco et al. 2013; Oh \& Yi 2012). Cane (2000) introduced the concept of a two-step FD, where the first step of the decrease is due to the shock and the second step is due to the ICME. Based on an extensive study of ICME-associated FDs at cosmic ray energies between $0.5-450 \mathrm{MeV}$, Richardson \& Cane (2011) conclude that shock and ICME effects are equally responsible for the FD. They also find that ICMEs that can be classified as magnetic clouds are usually involved in the largest of the FDs they studied. From now on, we will use the term CME to denote the CME near the Sun, as well as its counterpart observed from the Earth.

In this paper we have used FD data from the GRAPES-3 tracking muon telescope located at Ooty $\left(11.4^{\circ} \mathrm{N}\right.$ latitude, $76.7^{\circ} \mathrm{E}$ longitude, and $2200 \mathrm{~m}$ altitude) in south India. The GRAPES-3 muon telescope records the flux of muons in nine independent directions (labeled NW, N, NE, W, V, E, SW, S and SE), and the geomagnetic cutoff rigidity over this field of view varies from 12 to $42 \mathrm{GV}$. The details of this telescope are discussed in Hayashi et al. (2005), Nonaka et al. (2006), and Subramanian et al. (2009). Thus, the GRAPES-3 telescope observes the cosmic ray muon flux in nine independent directions with varying cutoff rigidities simultaneously. The high muon counting rate measured by the GRAPES-3 telescope results in extremely small statistical errors, allowing small changes in the 


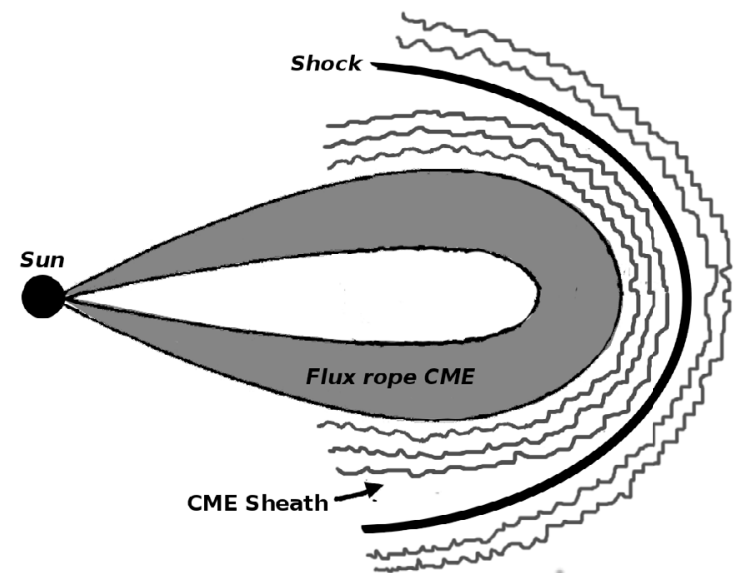

Fig. 1. A schematic of the CME-shock system. The CME is modeled as a flux rope structure. The undulating lines ahead of the shock denote MHD turbulence driven by the shock, while those in the CME sheath region denote turbulence in that region.

intensity of the cosmic ray flux to be measured with high precision. Thus a small drop $(\sim 0.2 \%)$ in the cosmic ray flux during a FD event can be reliably detected. This is possible even in the presence of the diurnal anisotropy of much larger magnitude $(\sim 1.0 \%)$, through a filtering technique described in Subramanian et al. (2009) (referred to hereafter as S09).

A schematic of the CME, which is assumed to have a fluxrope geometry (Vourlidas et al. 2012), together with the shock it drives, is shown in Fig. 1. The shock drives turbulence ahead of it, and there is also turbulence in the CME sheath region (e.g., Manoharan et al. 2000; Richardson \& Cane 2011).

Instead of treating the entire system shown in Fig. 1, which would be rather involved, we consider two separate models. The first, which we call the CME-only model, is one where the FD is assumed to be exclusively due to the CME, which is progressively populated by high energy cosmic rays as it propagates from the Sun to the Earth. The preliminary idea behind this model was first sketched by Cane et al. (1995) and was further developed in S09. However, the work we describe here addresses the multi-rigidity data, which is a major improvement over S09. We will describe several other salient improvements in the CMEonly model in subsequent sections. The second model, which we call the shock-only model, is one where the FD is assumed to arise only as a result of a propagating diffusive barrier, which is the shock driven by the CME (e.g., Wibberenz et al. 1998). The diffusive barrier would act as a shield for the Galactic cosmic ray flux, resulting in a lower cosmic ray density behind it. In treating these two models separately we aim to identify which is the dominant contributor to the observed FD, the CME, or the shock.

We identify FDs in the GRAPES-3 data that are associated with near-Earth magnetic clouds and possibly with the shocks driven by them. We describe our criteria for shortlisting events in the next section. We then test the extent to which each of the two models satisfies the multi-rigidity FD data from the GRAPES-3 muon telescope. In the subsequent analysis the use of cutoff rigidity rather than median rigidity is preferred for the following reason. The cutoff rigidity in a given direction represents the threshold rigidity of incoming cosmic rays and the magnitude of FD is a sensitive function of it. Instead, the median rigidity is comparatively insensitive to the magnitude of the FD.

\section{Criteria for shortlisting events}

\subsection{First and second shortlists: characteristics of the FD}

We have examined all FD events observed by the GRAPES-3 muon telescope from 2001 to 2004. We then shortlisted events that have magnitudes $>0.25 \%$ and possess a relatively clean profile comprising a sudden decrease followed by a gradual exponential recovery. While the figure of $0.25 \%$ might seem rather small by neutron monitor standards, we emphasize that the largest events observed with GRAPES-3 have magnitudes of $\sim 1 \%$. This list, which contains 72 events, is called shortlist 1 . We next correlate the events in shortlist 1 with lists of magnetic clouds near the Earth observed by the WIND and ACE spacecrafts (Huttunen et al. 2005; Lynch et al. 2003; A. Lara, priv. comm.) and select only those that can be connected reasonably well with a near-Earth magnetic cloud, and this set is labeled shortlist 2 (Table 1). The decrease minimum for most of the FD events in shortlist 2 lie between the start and the end of the nearEarth magnetic cloud.

\subsection{Third shortlist: $C M E$ velocity profile}

Since we are looking for FD events that are associated with shocks as well as CMEs, we examine the CME catalog ${ }^{1}$ for a near-Sun CME that can reasonably correspond to the near-Earth magnetic cloud that we associated with the FD in forming shortlist 2. In S09 it was assumed that the CME propagates with a constant speed from the Sun to the Earth. In this paper, we adopt a more realistic, two-step velocity profile described below.

The data from the LASCO coronograph aboard the SOHO spacecraft ${ }^{1} 1$ provide details about CME propagation up to a distance of $\approx 30 R_{\odot}$ from the Sun. We fit the velocity profile to the LASCO data points

$V_{1}=v_{\mathrm{i}}+a_{\mathrm{i}} t, \quad$ for $R(t) \leq R_{\mathrm{m}}$,

where $v_{\mathrm{i}}$ and $a_{\mathrm{i}}$ are the initial velocity and acceleration of CME, respectively, and $R_{\mathrm{m}}$ is the heliocentric distance at which the CME is last observed in the LASCO field of view. For distances $>R_{\mathrm{m}}$, we assume that the CME dynamics are governed exclusively by the aerodynamic drag it experiences due to momentum coupling with the ambient solar wind. For heliocentric distances $>R_{\mathrm{m}}$, we therefore use the widely used onedimensional differential equation to determine the CME velocity profile: (e.g., Borgazzi et al. 2009)

$m_{\mathrm{CME}} V_{2} \frac{\partial V_{2}}{\partial R}=\frac{1}{2} C_{\mathrm{D}} \rho_{\mathrm{sw}} A_{\mathrm{CME}}\left(V_{2}-V_{\mathrm{sw}}\right)^{2}, \quad R(t)>R_{\mathrm{m}}$,

where $m_{\mathrm{CME}}$ is the CME mass, $C_{\mathrm{D}}$ is the dimensionless drag coefficient, $\rho_{\mathrm{Sw}}$ is the solar wind density, $A_{\mathrm{CME}}=\pi R_{\mathrm{CME}}^{2}$ is the cross-sectional area of the CME, and $V_{\mathrm{sw}}$ is the solar wind speed. The boundary condition used is $V_{2}=v_{\mathrm{m}}$ at $R(t)=R_{\mathrm{m}}$. The CME mass $m_{\mathrm{CME}}$ is assumed to be $10^{15} \mathrm{~g}$ and the solar wind speed $V_{\mathrm{sw}}$ is taken to be equal to $450 \mathrm{~km} \mathrm{~s}^{-1}$. The solar wind density $\rho_{\mathrm{sw}}$ is given by the model of Leblanc et al. (1998). The composite velocity profile for the CME is defined by

$V_{\mathrm{CME}}= \begin{cases}V_{1}, & R(t) \leq R_{\mathrm{m}} \\ V_{2}, & R(t)>R_{\mathrm{m}}\end{cases}$

The total travel time for the CME is $\int_{R_{\mathrm{f}}}^{R_{\mathrm{f}}} \mathrm{d} R / V_{\mathrm{CME}}$, where $R_{\mathrm{i}}$ is the heliocentric radius at which the $\mathrm{CME}$ is first detected and

1 http://cdaw.gsfc.nasa.gov/CME_list/ 
Table 1. Selected events.

\begin{tabular}{lclllll}
\hline \hline Event & Magnitude (\%) & FD onset (UT) & FD min (UT) & MC start (UT) & MC stop (UT) & CME launch (UT) \\
\hline 2001 Apr. 11 & 2.86 & 2001 Apr. 11 12:00 & 2001 Apr. 12 18:00 & 2001 Apr. 11 23:00 & 2001 Apr. 12 18:00 & 2001 Apr. 10 05:30 \\
2001 Aug. 17 & 1.03 & 2001 Aug. 16 22:34 & 2001 Aug. 18 05:00 & 2001 Aug. 18 00:00 & 2001 Aug. 18 21:30 & 2001 Aug. 15 23:54 \\
2001 Sep. 29 & 2.44 & 2001 Sep. 29 12:43 & 2001 Oct. 1 01:00 & 2001 Sep. 29 16:30 & 2001 Sep. 30 11:30 & $\ldots$ not clear... \\
2001 Nov. 24 & 1.56 & 2001 Nov. 24 03:21 & 2001 Nov. 25 15:00 & 2001 Nov. 24 17:00 & 2001 Nov. 25 13:30 & 2001 Nov. 22 22:48 \\
2002 May 23 & 0.93 & 2002 May 23 02:10 & 2002 May 23 23:00 & 2002 May 23 21:30 & 2002 May 25 18:00 & $\ldots$ not clear... \\
2002 Sep. 7 & 0.97 & 2002 Sep. 7 14:52 & 2002 Sep. 8 13:00 & 2002 Sep. 7 17:00 & 2002 Sep. 8 16:30 & 2002 Sep. 5 16:54 \\
2002 Sep. 30 & 0.97 & 2002 Sep. 30 11:30 & 2002 Oct. 1 08:00 & 2002 Sep. 30 22:00 & 2002 Oct. 1 16:30 & $\ldots$ not clear... \\
2003 Nov. 20 & 1.16 & 2003 Nov. 20 10:48 & 2003 Nov. 24 04:00 & 2003 Nov. 22 06:10 & 2003 Nov. 22 06:50 & 2003 Nov. 18 08:50 \\
2004 Jul. 26 & 2.13 & 2004 Jul. 26 15:36 & 2004 Jul. 27 11:00 & 2004 Jul. 27 02:00 & 2004 Jul. 28 00:00 & 2004 Jul. 25 14:54 \\
\hline
\end{tabular}

Notes. Events that can clearly be associated with near-Sun CMEs and agree with the composite velocity profile (Sect. 2.2) have a CME launch time (near the Sun) mentioned next to them. These events form our final shortlist (shortlist 3). Magnitude: magnitude of FD in vertical direction. FD onset: time of FD onset in UT. FD minimum: time of FD minimum in UT. MC start: magnetic cloud start time in UT. MCstop: magnetic cloud stop time in UT. CME launch: time at which CME was first observed in the LASCO FOV.



Fig. 2. Velocity profile for the CME corresponding to the 2001 November 24 FD event. The CME was first observed in the LASCO FOV on 2001 November 22. The solid line shows the first stage governed by LASCO observations Eq. (1), where the CME is assumed to have a constant deceleration. The dashed line shows the second stage Eq. (2), where the CME is assumed to experience an aerodynamic drag characterized by a constant $C_{\mathrm{D}}$.

$R_{\mathrm{f}}$ is equal to $1 \mathrm{AU}$. We have used a constant drag coefficient $C_{\mathrm{D}}$ and adjusted its value so that the total travel time thus calculated matches the time elapsed between the first detection of the CME in the LASCO FOV and its detection as a magnetic cloud near the Earth. We have retained only those events for which it is possible to find a constant $C_{\mathrm{D}}$ and this criterion is satisfied. We have also eliminated magnetic clouds that could be associated with multiple CMEs. This defines our final shortlist, which we call shortlist 3 . The events that have an entry in the last column labeled CME launch in Table 1 comprise shortlist 3. We note that we have adjusted the parameter $C_{\mathrm{D}}$ so that the final CME speed obtained from Eq. (2) is close to the observed magnetic cloud speed near the Earth. It is usually not possible to find a $C_{\mathrm{D}}$ that will yield an exact match for the velocities and for the total travel times (e.g., Lara et al. 2011). Figure 2 shows an example of the composite velocity profile (given by Eqs. (1) and (2)) for a representative CME that was first observed in the LASCO FOV on 2001 November 22, and that resulted in a FD on 2001 November 24.

\section{Models for FDs}

We apply two different models to the FD events in Table 1; the first one is the CME-only model, which assumes that the FD owes its origin only to the CME. The second one is the shockonly model, which assumes that the FD is exclusively due to the shock, which is approximated as a diffusive barrier. Although both the shock and the CME are expected to contribute to the FD, our treatment seeks to determine which one of them is the dominant contributor at rigidities ranging from 14 to $24 \mathrm{GV}$. Before describing the models, we discuss the perpendicular diffusion coefficient.

\subsection{Perpendicular diffusion coefficient}

We use an isotropic perpendicular diffusion coefficient $\left(D_{\perp}\right)$ to characterize the penetration of cosmic rays across large scale, ordered magnetic fields. We envisage a CME, which has a flux rope structure, propagates outwards from the Sun, driving a shock ahead of it (see, e.g., Vourlidas et al. 2012). The flux rope CMEshock geometry is illustrated in Fig. 1. The CME sheath region between the CME and the shock is known to be turbulent (e.g., Manoharan et al. 2000) and it is well accepted that it has a significant role to play in determining FDs (Badruddin 2002; Yu et al. 2010). The isotropic perpendicular diffusion coefficient $D_{\perp}$ characterizes the penetration of cosmic rays through the ordered, compressed large-scale magnetic field near the shock, and across the ordered magnetic field of the flux rope CME. In diffusing across the shock, the cosmic rays are affected by the turbulence ahead of the shock, and in diffusing across the magnetic fields bounding the flux rope $\mathrm{CME}$, the cosmic rays are affected by the turbulence in the CME sheath region.

The subject of charged particle diffusion across field lines in the presence of turbulence is a subject of considerable research. Analytical treatments include the so-called classical scattering theory (e.g., Giacalone \& Jokipii 1999, and references therein), and the nonlinear guiding center theory (Matthaeus et al. 2003; Shalchi 2010) for perpendicular diffusion. Numerical treatments include Giacalone \& Jokipii (1999), Casse et al. (2002), Candia $\&$ Roulet (2004) and Tautz \& Shalchi (2011). For our purposes, we seek a concrete, usable prescription for the $D_{\perp}$ that can incorporate as many observationally determined quantities as possible. We find that the analytical fits to extensive numerical simulations provided by Candia \& Roulet (2004), best suit our requirements. We note, in particular, that their results not only reproduce the standard results of Giacalone \& Jokipii (1999) and Casse et al. (2002) but also extend the regime of validity to include strong turbulence and high rigidities. Our approach is similar to that of Effenberger et al. (2012), who adopt empirical expressions for the perpendicular diffusion coefficients. It should be mentioned, however, that they allow for the possibility of anisotropic perpendicular diffusion. We treat the case 
of radial diffusion across the largely azimuthal magnetic fields bounding a flux rope CME structure, and we therefore need only one (isotropic) perpendicular diffusion coefficient $\left(D_{\perp}\right)$.

In the formulation of Candia \& Roulet (2004), the perpendicular diffusion coefficient $D_{\perp}$ is a function of the quantity $\rho$ (which is closely related to the rigidity and indicates how tightly the proton is bound to the magnetic field) and the level of turbulence $\sigma^{2}$. Our characterization of $D_{\perp}\left(\rho, \sigma^{2}\right)$ follows that of S 09 , who adopt the analytical fits to Monte Carlo simulations of particle transport in turbulent magnetic fields given by Candia \& Roulet (2004). The quantity $\rho$ is related to the rigidity $R_{\mathrm{g}}$ by

$\rho=\frac{R_{\mathrm{L}}}{L_{\max }}=\frac{R_{\mathrm{g}}}{B_{0} L_{\max }}$

where $R_{\mathrm{L}}$ is the Larmor radius and $B_{0}$ is the strength of the relevant large-scale magnetic field. For the CME-only model, $B_{0}$ refers to the large-scale magnetic field bounding the CME, and for the shock-only model, it refers to the enhanced largescale magnetic field at the shock. In writing second step in Eq. (4), we have related the Larmor radius to the rigidity $R_{\mathrm{g}}$ by

$R_{\mathrm{L}}(t)=\frac{R_{\mathrm{g}}}{B_{0}}$

For the CME-only model, we adopt $L_{\max }=2 R(T)$, where $R(T)$ is the radius of the near-Earth magnetic cloud. This is in contrast with S09, where $L_{\max }$ was taken to be $10^{6} \mathrm{~km}$, which is the approximate value for the outer scale of the turbulent cascade in the solar wind. For the shock-only model, on the other hand, we assume that $L_{\max }$ is equal to $1 \mathrm{AU}$.

The turbulence level $\sigma^{2}$ is defined (as in Candia \& Roulet 2004, and S09) to be

$\sigma^{2} \equiv \frac{\left\langle B_{\mathrm{r}}{ }^{2}\right\rangle}{B_{0}{ }^{2}}$

where $B_{\mathrm{r}}$ is the fluctuating part of the turbulent magnetic field and the angular braces denote an ensemble average.

For the sake of completeness, we reproduce the full expression for the isotropic perpendicular diffusion coefficient that we use in this work. It is the same as the one used in S09, and is taken from Candia \& Roulet (2004). The diffusion coefficient due to scattering of particles along the mean magnetic field $D_{\|}$ is given by

$D_{\|}=c L_{\max } \rho \frac{N_{\|}}{\sigma^{2}} \sqrt{\left(\frac{\rho}{\rho_{\|}}\right)^{2(1-\gamma)}+\left(\frac{\rho}{\rho_{\|}}\right)^{2}}$,

where $c$ is the speed of light and the quantities $N_{\|}, \gamma$ and $\rho_{\|}$are constants specific for different kinds of turbulence. The perpendicular diffusion coefficient $\left(D_{\perp}\right)$ is related to the parallel coefficient $\left(D_{\|}\right)$by

$\frac{D_{\perp}}{D_{\|}}= \begin{cases}N_{\perp}\left(\sigma^{2}\right)^{a_{\perp}}, & \rho \leq 0.2 \\ N_{\perp}\left(\sigma^{2}\right)^{a_{\perp}}\left(\frac{\rho}{0.2}\right)^{-2}, & \rho>0.2 .\end{cases}$

The quantities $N_{\perp}$ and $a_{\perp}$ are constants specific to different kinds of turbulent spectra. In this work, we assume the Kolmogorov turbulence spectrum in our calculations. We use $N_{\|}=1.7, \gamma=$ $5 / 3, \rho_{\|}=0.20, N_{\perp}=0.025$, and $a_{\perp}=1.36$ (Table 1, Candia $\&$ Roulet 2004). Equation (7) together with Eq. (8) defines the isotropic perpendicular diffusion coefficient we use in this work.



Fig. 3. An illustration depicting a flux rope CME expanding and propagating away from the Sun. High-energy Galactic cosmic rays diffuse into the CME across its bounding magnetic field.

\subsection{CME-only model}

The basic features of the CME-only model are similar to the one used in S09 and here we only highlight the areas where there are significant differences from S09. There are practically no highenergy Galactic cosmic rays inside the CME when it starts out near the Sun. The cosmic rays diffuse into it from the surroundings via perpendicular diffusion across the closed magnetic field lines as it propagates through the heliosphere as shown schematically in Fig. 3. Near the Earth, the difference between the (relatively lower) cosmic ray proton density inside the CME and that in the ambient medium appears as the FD. We then obtain an estimate of the cosmic ray proton density inside the CME produced by the cumulative effect of diffusion.

The flux $F$ of protons diffusing into the $\mathrm{CME}$ at a given time depends on the isotropic perpendicular diffusion coefficient $D_{\perp}$ and the density gradient $\partial N_{\mathrm{a}} / \partial r$, and can be written as

$F\left(\mathrm{~cm}^{-2} \mathrm{~s}^{-1}\right)=D_{\perp} \frac{\partial N_{\mathrm{a}}}{\partial r}$.

As mentioned earlier, the $D_{\perp}$ characterizes diffusion across the (largely closed) magnetic fields bounding the CME, and $N_{\mathrm{a}}$ is the ambient density of high energy protons. The total number of cosmic ray protons that will have diffused into the CME after a time $T$ is related to the diffusing flux by

$U_{\mathrm{i}}=\int_{0}^{T} A(t) F(t) \mathrm{d} t=\int_{0}^{T} D_{\perp} A(t) \frac{\partial N_{\mathrm{a}}}{\partial r} \mathrm{~d} t$,

where $\mathrm{A}(\mathrm{t})$ is the cross-sectional area of the CME at a given time $t$. According to our convention, the CME is first observed in the LASCO FOV at $t=0$ and reaches the Earth at $t=T$. The ambient density gradient $\partial N_{\mathrm{a}} / \partial r$ is approximated by the expression

$\frac{\partial N_{\mathrm{a}}}{\partial r} \simeq \frac{N_{\mathrm{a}}}{L}$

which is significantly different from that used in S09, where $L$ is the gradient lengthscale. Observations of the density gradient lengthscale $L$ exist only for a few rigidities. We use the observations of Heber et al. (2008), who quote a value of $L^{-1}=$ $4.7 \% \mathrm{AU}^{-1}$ for $1.2 \mathrm{GV}$ protons. We take this as our reference value. In order to calculate $L$ for other rigidities (in the 14-24 GV range that we use here), we assume that $L \propto R_{\mathrm{L}}^{1 / 3}$. This is broadly consistent with the observation (De Simone et al. 2011) that the density gradient lengthscale is only weakly dependent on rigidity. For a given rigidity, we also need the value 
of $L$ all the way from the Sun to the Earth. In order to calculate this, we recognize that $L$ near the CME/magnetic cloud will not be the same as its value in the ambient solar wind. We adopt $L \propto B_{\mathrm{a}} / B_{\mathrm{MC}}$, where $B_{\mathrm{MC}}$ is the large-scale magnetic field bounding the CME and $B_{\mathrm{a}}$ is the (weaker) magnetic field in the ambient medium outside the CME. Furthermore, while $B_{\mathrm{MC}}$ varies according to Eq. (12) below, the ambient field $B_{\text {a }}$ of the Parker spiral in the ecliptic plane varies inversely with heliocentric distance.

We assume that the magnetic flux associated with the CME is frozen-in with it as it propagates. In other words, the product of the CME magnetic field and the CME cross-sectional area remains constant. This is a fairly well-founded assumption (e.g., Kumar \& Rust 1996; Subramanian \& Vourlidas 2007). One can therefore relate the CME magnetic field $B_{0}(t)$ at a given time $t$ to the value $B_{\mathrm{MC}}$ measured in the near-Earth magnetic cloud by

$B_{0}(t)=B_{\mathrm{MC}}\left[\frac{R(T)}{R(t)}\right]^{2}$,

where $R(T)$ is the radius of the magnetic cloud observed from the Earth and $R(t)$ is its radius at any other time $t$ during its passage from the Sun to the Earth. The CME radius $R(t)$ and $R(T)$ are related via Eq. (15) below. We emphasize that the magnetic field in Eq. (12) refers to the magnetic field bounding the CME, and not the ambient magnetic field outside it.

We model the CME as an expanding cylindrical flux rope whose length increases with time as it propagates outwards. Its cross-sectional area at time $t$ is

$A(t)=2 \pi L(t) R(t)$,

where $L(t)$ is the length of the flux-rope cylinder at time $t$, and is related to the height $H(t)$ of the CME above the solar limb via

$L(t)=\pi H(t)$.

We note that Eq. (14) differs from the definition used in S09 by a factor of 2 . We assume that the CMEs expand in a selfsimilar manner as they propagate outwards. The three dimensional flux rope fittings to CMEs in the $\sim 2-20 R_{\odot}$ field of view using SECCHI/STEREO data validate this assumption (e.g., Poomvises et al. 2010). The self-similar assumption means that the radius of the $R(t)$ of the flux rope is related to its heliocentric height $H(t)$ by

$\frac{R(t)}{H(t)}=\frac{R(T)}{H(T)}$,

where $H(T)$, the heliocentric height at time $T$, is $=1$ AU by definition, and $R(T)$ is the measured radius of the magnetic cloud from the Earth.

As mentioned earlier, we consider a two-stage velocity profile for CME propagation, expressed by Eqs. (1) and (2); this is substantially different from the constant speed profile adopted in S09.

Using Eqs. (11), (13) and (14) in (10), we get the expression for the total number of protons inside the CME when it arrives on the Earth

$U_{\mathrm{i}}=\int_{0}^{T} 2 \pi L(t) R(t) D_{\perp} \frac{N_{\mathrm{a}}}{\kappa R_{\mathrm{L}}(t)^{0.33}} \mathrm{~d} t$.

The cosmic ray density inside the CME when it arrives on the Earth is

$N_{\mathrm{i}}=\frac{U_{\mathrm{i}}}{\pi R(T)^{2} L(T)}$,

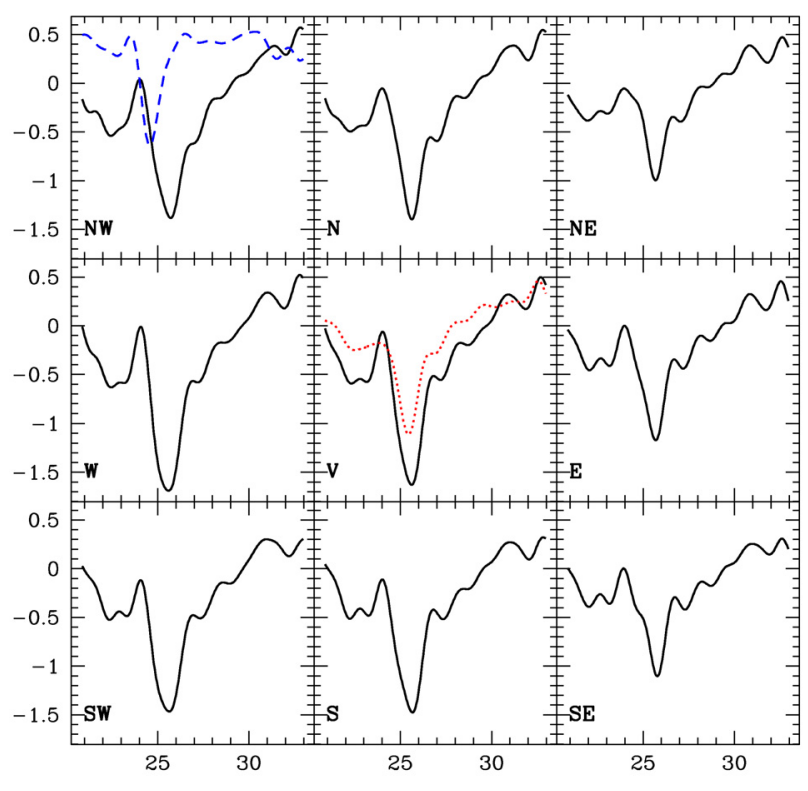

Fig. 4. Muon flux along the nine directions is shown for the FD on 2001 November 24. The fluxes are shown as percentage deviations from mean values. The solid black lines show the data after applying a lowpass filter (S09). The blue dashed line in the first panel shows the magnetic field observed in-situ by spacecraft. The magnetic field data are inverted (i.e., magnetic field peaks appear as troughs) and are scaled to fit in the panel. The red dotted line in the middle panel shows Tibet neutron monitor data scaled down by a factor of 3 to fit in the panel.

where $L(T)$ and $R(T)$ are the length and cross-sectional radius of the CME, respectively, at time $T$, when it reaches the Earth. When the CME arrives on the Earth, the relative difference between the cosmic ray density inside the CME and the ambient environment is manifested as the FD, whose magnitude $M$ can be written as

$M=\frac{N_{\mathrm{a}}-N_{\mathrm{i}}}{N_{\mathrm{a}}}=\frac{\Delta N}{N_{\mathrm{a}}}$.

We compare the value of the FD magnitude $M$ predicted by Eq. (18) with observations in Sect. 4.

An example of an FD event observed in all 9 bins of GRAPES-3 is shown in Fig. 4. The $x$-axis is the time in days starting from 2001 November 1 and the $y$-axis gives the percentage deviation of the muon flux from the pre-event mean. The magnitude $M$ of the FD for a given rigidity bin is the difference between the pre-event cosmic ray intensity and the intensity at the minimum of the FD.

\subsection{Shock-only model}

In this approach, we assume that the FD is caused exclusively by the shock, which is modeled as a propagating diffusive barrier. The expression for the magnitude of the FD according to this model is (Wibberenz et al. 1998)

$M \equiv \frac{U_{\mathrm{a}}-U_{\text {shock }}}{U_{\mathrm{a}}}=\frac{\Delta U}{U_{\mathrm{a}}}=\frac{V_{\text {sw }} L_{\text {shock }}}{D_{\perp}{ }^{\mathrm{a}}}\left(\frac{D_{\perp}{ }^{\mathrm{a}}}{D_{\perp}{ }^{\text {shock }}}-1\right)$,

where $U_{\mathrm{a}}$ is the ambient cosmic ray density and $U_{\text {shock }}$ is that inside the shock, $D_{\perp}{ }^{\mathrm{a}}$ is the ambient isotropic perpendicular diffusion coefficient and $D_{\perp}$ shock is that inside the shock, $V_{\mathrm{sw}}$ is the solar wind velocity, and $L_{\text {shock }}$ is the shock sheath thickness. For each shock event, we examine the magnetic field data from the 

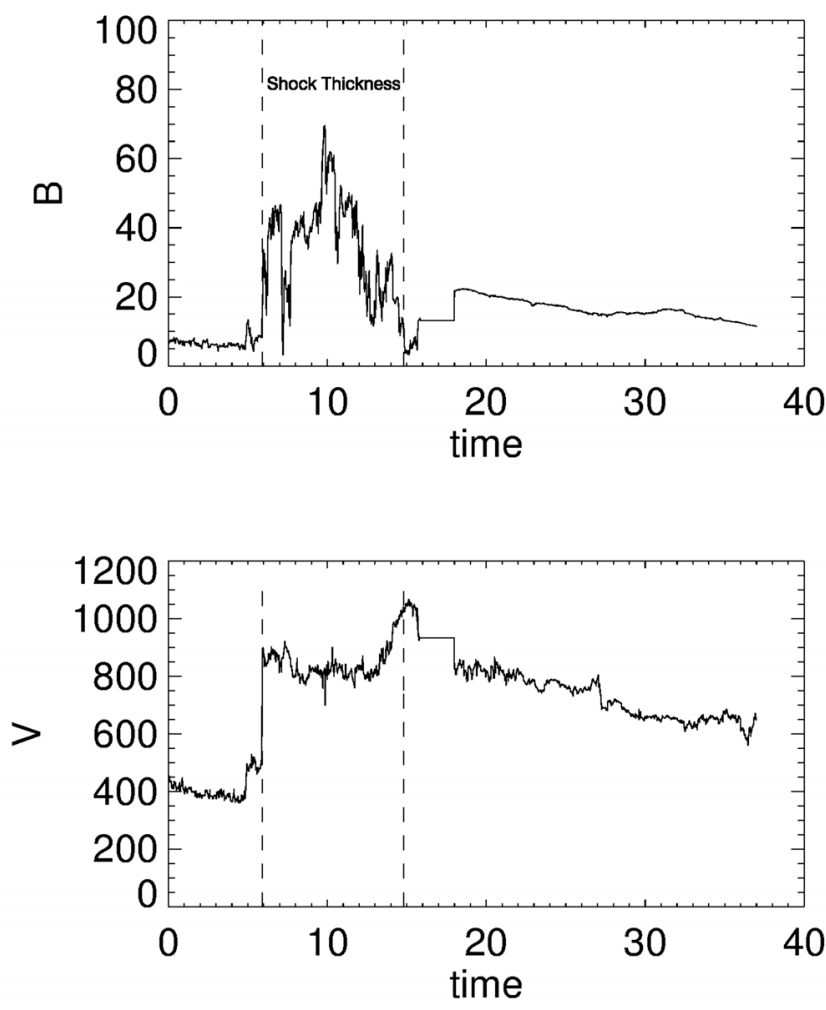

Fig. 5. Interplanetary magnetic field and solar wind speed from the day 2001 November 24, The shock sheath thickness is computed by multiplying the time interval inside the dotted lines by the solar wind speed.

ACE and WIND spacecrafts and estimate the shock sheath thickness $L_{\text {shock }}$ to be the spatial extent of the magnetic field enhancement. An example is shown in Fig. 5.

In computing $D_{\perp}{ }^{\mathrm{a}}$ and $D_{\perp}{ }^{\text {shock }}$ we need to use different values for the proton rigidity $\rho$ for the ambient medium and in the shock sheath; they are related to the proton rigidity $R_{\mathrm{g}}$ by

$\rho^{\mathrm{a}}=\frac{R_{\mathrm{g}}}{B_{0}{ }^{\mathrm{a}} L_{\text {shock }}}$

$\rho^{\text {shock }}=\frac{R_{\mathrm{g}}}{B_{0}^{\text {shock }} L_{\text {shock }}}$,

where $B_{0}^{\mathrm{a}}$ is the ambient magnetic field and $B_{0}^{\text {shock }}$ is the magnetic field inside the shock sheath.

\section{Results}

In this section we first describe various parameters needed for the CME-only and the shock-only models that are derived from observations. Using these parameters, we examine whether the notion of cosmic ray diffusion is valid for each model. Using the observationally determined parameters, we can determine which of the two models best reproduces the observed FD magnitude in each rigidity bin.

\subsection{Observationally derived parameters}

Table A. 2 contains the observationally determined parameters for each of the CMEs and their corresponding shocks in the final shortlist (Table 1).

The quantity First obs denotes the time (in UT) when the CME was first observed in the LASCO FOV, while $R_{\text {first }}$ is the
Table 2. Minimum $\chi^{2}$ values for the CME-only model fits to GRAPES-3 data.

\begin{tabular}{lc}
\hline \hline Event & $\chi^{2}$ \\
\hline 2001 April 11 & 11.1 \\
2001 August 17 & 1.96 \\
2001 November 24 & 2.46 \\
2002 September 7 & 25.5 \\
2003 November 20 & 3.66 \\
2004 July 26 & 27.5 \\
\hline
\end{tabular}

distance (in units of $R_{\odot}$ ) at which CME was first observed in LASCO FOV and $R_{\text {last }}$ is the distance at which the CME was last observed in LASCO FOV. The quantity $V_{\exp }$ is the speed of CME at $R_{\text {last }}$ (in $\mathrm{km} \mathrm{s}^{-1}$ ) and $a_{\mathrm{i}}$ is the acceleration of CME in the LASCO FOV (in $\mathrm{m} \mathrm{s}^{-2}$ ). The quantity $C_{\mathrm{D}}$ is the (constant) dimensionless drag coefficient used for the velocity profile (Sect. 2.2). The quantities MC start and MC end denote the start and end times of the magnetic cloud in UT. The quantity $V_{\mathrm{sw}}^{\mathrm{MC}}$ is solar wind speed at the Earth (in $\mathrm{km} \mathrm{s}^{-1}$ ) just ahead of the arrival of the magnetic cloud, and $R_{\mathrm{MC}}$ is the radius of the magnetic cloud (in $\mathrm{km}$ ). The quantity $B_{\mathrm{MC}}$ is the peak magnetic field inside the magnetic cloud (in nT). The quantity $T_{\text {total }}$ is the SunEarth travel time (in h) taken by the CME to travel from Sun to Earth. The quantity Shock arrival denotes the time (in UT) when the shock is detected near the Earth. The quantities $B^{\mathrm{a}}$ and $B^{\text {shock }}$ represent the magnetic fields (in $\mathrm{nT}$ ) in the ambient solar wind and inside the shock sheath region, respectively (see Fig. 5 for an example). The quantity $V_{\mathrm{sw}}^{\text {shock }}$ represents the near-Earth shock speed in $\mathrm{km} \mathrm{s}^{-1}$.

Table A.1 contains details of the FDs associated with each of the CMEs in the final shortlist. The magnitude of the FD in a given rigidity bin is the difference between the pre-event intensity of the cosmic rays and the intensity at the minimum of the FD. It also contains the onset and the time of minimum, and the magnitude of the decrease in each bin (together with the corresponding cutoff rigidity) for each of the FD events in our final shortlist.

\subsection{Fitting the CME-only and shock-only models to multi-rigidity FD data}

Using the observational parameters listed in Table A.2, we have computed the magnitude of the FD using the CME-only (Sect. 3.2) and shock-only models (Sect. 3.3). The only free parameter in our model is the ratio of the energy density in the random magnetic fields to that in the large scale magnetic field $\sigma^{2} \equiv\left\langle B_{\text {turb }}{ }^{2} / B_{0}{ }^{2}\right\rangle$. Figure 6 shows the best fits of the CMEonly model to the multi-rigidity data. The only free parameter in the model is $\sigma^{2} \equiv\left\langle B_{\text {turb }}{ }^{2} / B_{0}^{2}\right\rangle$, and the best fit is chosen by minimizing the $\chi^{2}$ with respect to $\sigma^{2}$. For each FD event, the * symbols denote the observed FD magnitude for a given rigidity bin. The dashed line denotes the FD magnitude predicted by the CME-only model. We define the chi-square statistic as

$\chi_{=}^{2} \sum_{\mathrm{i}} \frac{\left(E_{\mathrm{i}}-D_{\mathrm{i}}\right)^{2}}{v a r_{\mathrm{i}}}$,

where $E_{\mathrm{i}}$ is the value predicted by the theoretical model, $D_{\mathrm{i}}$ is the corresponding GRAPES-3 data point, and $\operatorname{var}_{i}$ is the variance for the corresponding data points. The $\chi^{2}$ values obtained after minimizing with respect to $\sigma^{2}$ are listed in Table 2 .

The entries in the second column $\sigma_{\mathrm{MC}}$ in Table 3 denote the square roots of the turbulence parameter $\sigma^{2}$ that we have used for 


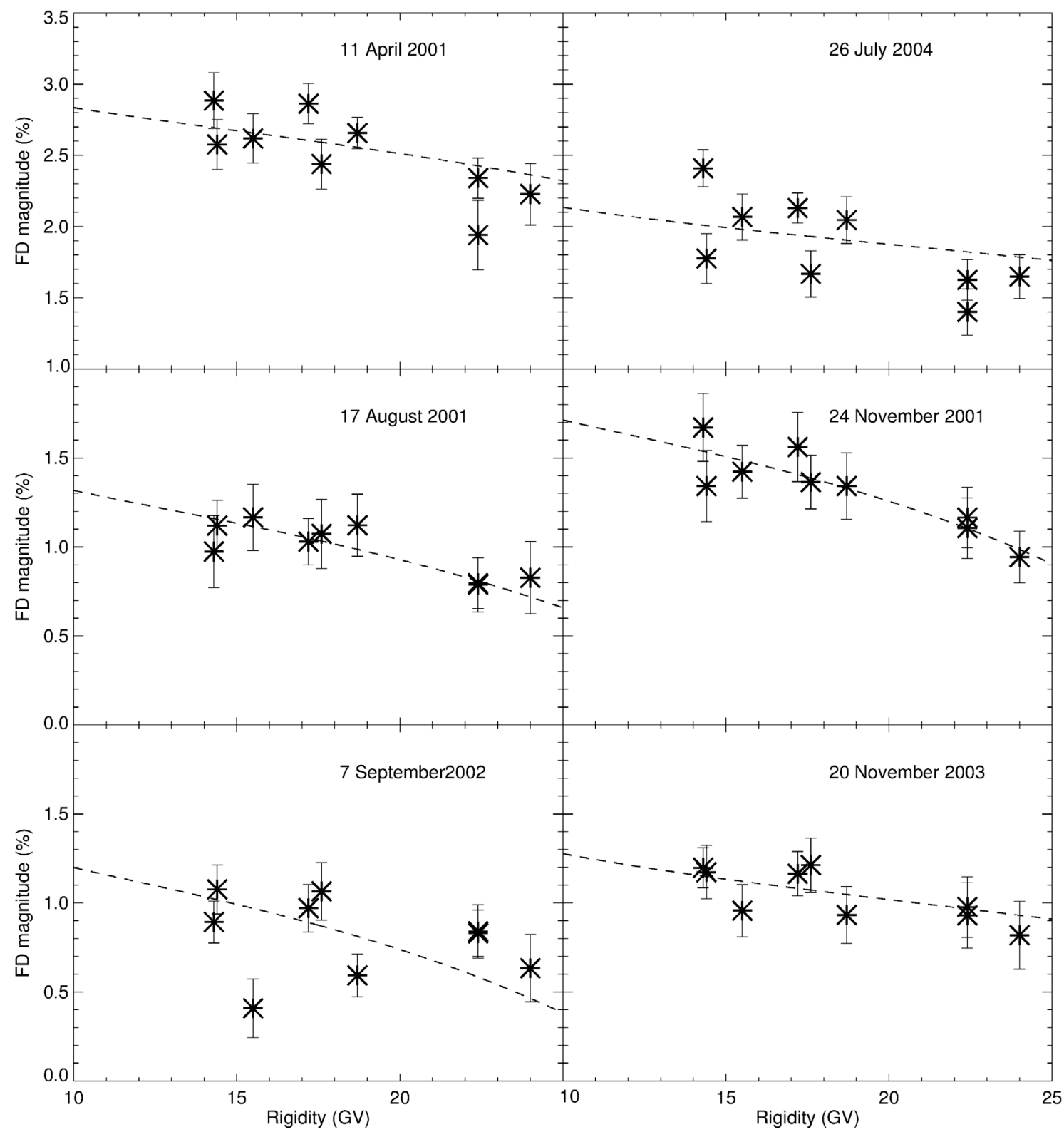

Fig. 6. FD magnitude observed with GRAPES-3 (* symbols). The dashed line is obtained using the CME-only model.

Table 3. Turbulence levels in the sheath region required by the models.

\begin{tabular}{lcc}
\hline \hline Event & $\begin{array}{c}\text { CME-only model } \\
\sigma_{\mathrm{MC}}\end{array}$ & $\begin{array}{c}\text { shock-only model } \\
\sigma_{\text {Shock }}\end{array}$ \\
\hline 2001 April 11 & $9.4 \%$ & $100 \%$ \\
2001 August 17 & $13 \%$ & $180 \%$ \\
2001 November 24 & $28 \%$ & $400 \%$ \\
2002 September 7 & $13 \%$ & $100 \%$ \\
2003 November 20 & $6.7 \%$ & $400 \%$ \\
2004 July 26 & $46 \%$ & $200 \%$ \\
\hline
\end{tabular}

the model fits for each event. These values represent the level of turbulence in the sheath region immediately ahead of the CME, through which the cosmic rays must traverse in order to diffuse into the CME. By comparison, the value of $\sigma$ for the quiescent solar wind ranges from 6 to 15\% (Spangler 2002). Evidently, the CME-only model implies that the sheath region ahead of the CME is only a little more turbulent than the quiescent solar wind, except for the 2004 July 26 event, where the speed of $\mathrm{CME}$ at the Earth was much higher than that for the other events.

We have carried out a similar exercise for the shock-only model (Sect. 3.3). For each event, we have used the observationally obtained parameters pertaining to the shock listed in Table A.2. Since this model needs the turbulence levels in both the ambient medium and the shock sheath region to be specified, we have assumed that the turbulence level inside the shock sheath region is twice that in the ambient medium. We find that it is not possible to fit the shock-only model to the multi-rigidity data using values for the turbulence parameter that are reasonably close to that in the quiescent solar wind. For each event, the column labeled $\sigma_{\text {Shock }}$ in Table 3 denotes the turbulence level in the shock sheath region that is required to obtain a reasonable fit to the data. Clearly, these values are an order of magnitude higher than those observed in the quiet solar wind. 


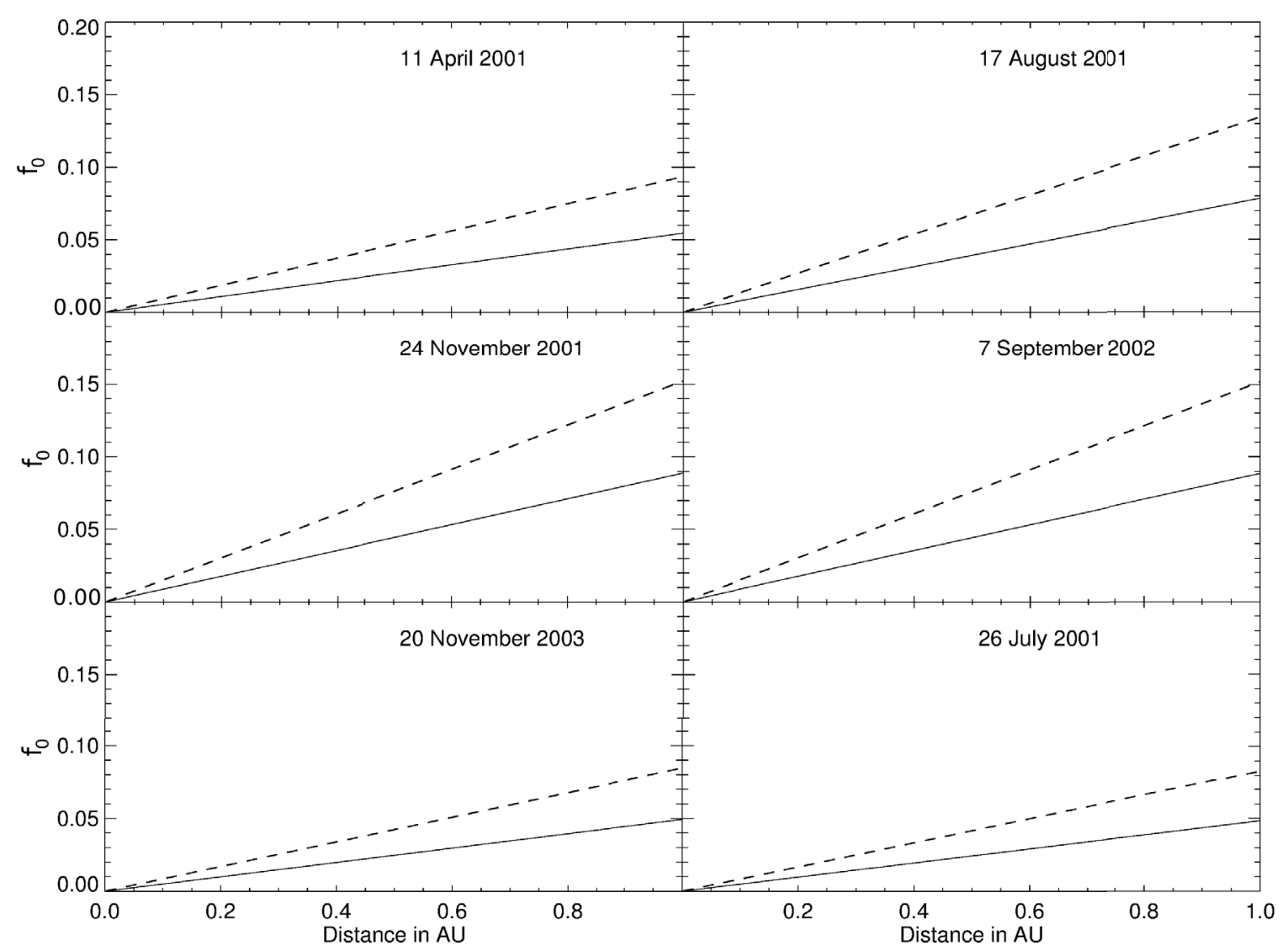

Fig. 7. $f_{0} \equiv R_{\mathrm{L}} / R$ versus distance as a CME propagates from the Sun to the Earth. The dashed and continuous lines represent $24 \mathrm{GV}$ and $12 \mathrm{GV}$ protons, respectively.

\section{3. $R_{L} / R_{C M E}$ for CME-only model}

Kubo \& Shimazu (2010) have simulated the process of cosmic ray diffusion into an ideal flux rope CME in the presence of MHD turbulence. They find that, if the quantity $f_{0}(t) \equiv R_{\mathrm{L}}(t) / R(t)$ is small, cosmic ray penetration into the flux rope is dominated by diffusion via turbulent irregularities. Other effects such as gradient drift due to the curvature of the magnetic field are unimportant under these conditions. Figure 7 shows the quantity $f_{0}(t) \equiv R_{\mathrm{L}}(t) / R(t)$ for 12 and $24 \mathrm{GV}$ protons, for each of the CMEs in our final shortlist (Table 1). The Larmor radius $R_{\mathrm{L}}(t)$ is defined by Eqs. (5) and (12) and the CME radius $R(t)$ is defined in Eq. (15). Clearly, $f_{0} \ll 1$ all through the Sun-Earth passage of the CMEs, and this means that the role of MHD turbulence in aiding penetration of cosmic rays into the flux rope structure is expected to be important.

\section{Summary}

Our main aim in this work is to determine whether FDs due to cosmic rays of rigidities ranging from 14 to $24 \mathrm{GV}$ are caused primarily by the CME, or by the shock associated with it. We examine this question in the context of multi-rigidity FD data from the GRAPES-3 instrument. We use a carefully selected sample of FD events from GRAPES-3 that are associated with both CMEs and shocks.

We consider two models: the CME-only model (Sect. 3.2) and the shock-only model (Sect. 3.3). In the CME-only model, we envisage the $\mathrm{CME}$ as an expanding bubble bounded by large-scale magnetic fields. The CME starts out near the Sun with practically no high energy cosmic rays inside it. As it travel towards the Earth, high-energy cosmic rays diffuse into the CME across the large-scale magnetic fields bounding it. The diffusion coefficient is a function of the rigidity of the cosmic ray particles as well as the level of MHD turbulence in the vicinity of the CME (the sheath region). Despite the progressive diffusion of cosmic rays into it, the cosmic ray density inside the CME is still lower than the ambient density when it reaches the Earth. When the CME engulfs the Earth, this density difference causes the FD observed by cosmic ray detectors. In the shock-only model, we consider the shock as a propagating diffusive barrier. It acts as an umbrella against cosmic rays, and the cosmic ray density behind the umbrella is lower than that ahead of it.

We have obtained a list of FD events observed by the GRAPES-3 instrument using the shortlisting criteria described in Sect. 2. For each of these shortlisted events, we have used observationally derived parameters listed in Table A.2 for both the models. The only free parameter was the level of MHD turbulence (defined as the square root of the energy density in the turbulent magnetic fluctuations to that in the large-scale magnetic field) in the sheath region.

\section{Conclusions}

Figure 6 shows the results of the CME-only model fits to multirigidity data for each of the shortlisted events. For the shock-only model, we use the turbulence level in the shock sheath region as the free parameter. Table 3 summarizes the values of these 
turbulence levels that we have used for each of the FD events in the final shortlist. These values may be compared with the estimate of 6 to $15 \%$ for the turbulence level in the quiescent solar wind (Spangler 2002). We thus find that a good model fit using the CME-only model requires a turbulence level in the sheath region that is typically only a little higher than that in the quiescent solar wind, which is generally consistent with observations. On the other hand, a good fit using the shock-only model demands a turbulence level in the shock sheath region that is often an order of magnitude higher than that in the quiet solar wind, which is somewhat unrealistic. The results summarized in Table 3, imply that, for FDs involving protons of rigidities ranging from 14 to $24 \mathrm{GV}$, the CME-only model is a viable one, while the shock-only model is not. Given the remarkably good fits to multi-rigidity data (Fig. 6), the reasonable turbulence levels in the sheath region demanded by the CME-only model (Table 3) and because the FD minima usually occur well within the magnetic cloud (Table A.1), we conclude that CMEs are the dominant contributors to the FDs observed by the GRAPES-3 experiment.

Acknowledgements. K.P. Arunbabu acknowledges support from a Ph.D. studentship at IISER Pune. P. Subramanian acknowledges partial support from the RESPOND program administered by the Indian Space Research Organization. We thank Alejandro Lara for useful discussions and the referee K. Scherer for several critical and helpful suggestions. We thank D. B. Arjunan, A. Jain, the late S. Karthikeyan, K. Manjunath, S. Murugapandian, S. D. Morris, B. Rajesh, B. S. Rao, C. Ravindran, and R. Sureshkumar for their help in the testing, installation, and operating the proportional counters and the associated electronics and during data acquisition. We thank G. P. Francis, I. M. Haroon, V. Jeyakumar, and K. Ramadass for their help in the fabrication, assembly, and installation of various mechanical components and detectors.

\section{References}

Badruddin 2002, Ap\&SS, 281, 651

Badruddin, Yadav, R. S., \& Yadav, N. R. 1986, Sol. Phys., 105, 413

Blanco, J. J., Catalan, E., Hidalgo, M. A., Medina, J., \& Rodiguez-Pacheco, J. 2013, Sol. Phys., DOI: 10.1007/s11207-013-0256-1

Bothmer, V., \& Schwenn, R. 1998, Ann. Geophys., 16, 1

Borgazzi, A., Lara, A., Echer, E., et al. 2009, A\&A, 498, 885

Burlaga, L. F., Sittler, E., Mariani, F., \& Schwenn, R. 1981, J. Geophys. Res., 86,6673
Candia, J., \& Roulet, E. 2004, J. Cosmol. Astropart. Phys., 10, 7

Cane, H. V. 2000, Space Sci. Rev., 93, 55

Cane, H. V., Richardson, I. G., \& Wibberenz, G. 1995, Proc. 24th Int. Cosmic Ray Conf., 4, 377

Casse, F., Lemoine, M., \& Pelletier, G. 2002, Phys. Rev. D, 65, 023002

de Simone, N., di Felice, V., Gieseler, J., et al. 2011, ASTRA, 7, 425

Effenberger, F., Fichtner, H., Scherer, K., et al. 2012, ApJ, 750, 108

Giacalone, J., \& Jokipii, J. R. 1999, ApJ, 520, 204

Hayashi, Y., Aikawa, Y., Gopalakrishnan, N. V., et al. 2005, Nucl. Instrum. Meth. A, 545, 643

Heber, B., Gieseler, J., Dunzlaff, P., et al. 2008, ApJ, 689, 1443

Huttunen, K. E. J., Schwenn, R., Bothmer, V., \& Koskinen, H. E. J. 2005, Ann. Geophys., 23, 625

Kubo, Y., \& Shimazu, H. 2010, ApJ, 720, 853

Kuwabara, T., Bieber, J. W., Evensen, P., et al. 2009, J. Geophys. Res., 114, 05109

Lara, A., Flandes, A., Borgazzi, A., \& Subramanian, P. 2011, J. Geophys. Res., 116, CiteID A12102

Leblanc, Y., Dulk, G. A., Bougeret, J.-L., et al. 1998, Sol. Phys., 183, 165

Lockwood, J. A., Webber, W. R., \& Debrunner, H. 1991, J. Geophys. Res., 96, 11587

Lynch, B. J., Zurbuchen, T. H., Fisk, L. A., \& Antiochos, S. K. 2003, J. Geophys. Res., 108, 1239

Manoharan, P. K., Kojima, M., Gopalswamy, N., et al. 2000, ApJ, 530, 1061

Matthaeus, W. S., Qin, G., Bieber, J. W., \& Zank, G. P. 2003, ApJ, 590, L53

Nonaka, T., Hayashi, Y., Ito, N., et al. 2006, Phys. Rev. D, 74, 052003

Oh, S. Y., \& Yi, Y. 2012, Sol. Phys., 280, 197

Poomvises, W., Zhang, J., Olmedo, O., 2010, ApJ, 717, L159

Reames, D. V., Kahler, S. W., \& Tylka, A. J. 2009, ApJ, 700, 196

Richardson, I. G., \& Cane , H. V. 2011, Sol. Phys., 270, 609

Sanderson, T. R., Beeck, J., Marsden, G. R., et al. 1990, Proc. 21st Int. Cosmic Ray Conf., 6, 251

Shalchi, A. 2010, ApJ, 720, L127

Schwenn, R., Dal Lago, A., Huttunen, E., \& Gonzalez, W. D. 2005, Ann. Geophys., 23, 1033

Spangler, S. R. 2002, ApJ, 576, 997

Subramanian, P., \& Vourlidas, A. 2007, A\&A, 467, 685

Subramanian, P., Antia, H. M., Dugad, S. R., et al. 2009, A\&A, 494, 1107

Tautz, R. C., \& Shalchi, A. 2011, ApJ, 735, 92

Vourlidas, A., Lynch, B. J., Howard, R. A., \& Li, Y. 2012, Sol. Phys., 192V: DOI: $10.1007 / \mathrm{s} 11207-012-0084-8$

Wang Y., Zhou, G., Ye, P., Wang, S., \& Wang, J. 2006, ApJ, 651, 1245

Wibberenz, G., le Roux, J. A., Potgieter, M. S., \& Bieber, J. W. 1998, Space Sci. Rev., 83, 309

Yu, X. X., Lu, H., Le, G. M., \& Shi, F., 2010, Sol. Phys., 263, 223

Zhang, G., \& Burlaga, L. F. 1988, J. Geophys. Res., 93, 2511

Pages 10 to 11 are available in the electronic edition of the journal at http://www . aanda. org 


\section{Appendix A: Tables}

Table A.1. Derived parameters of FD for events.

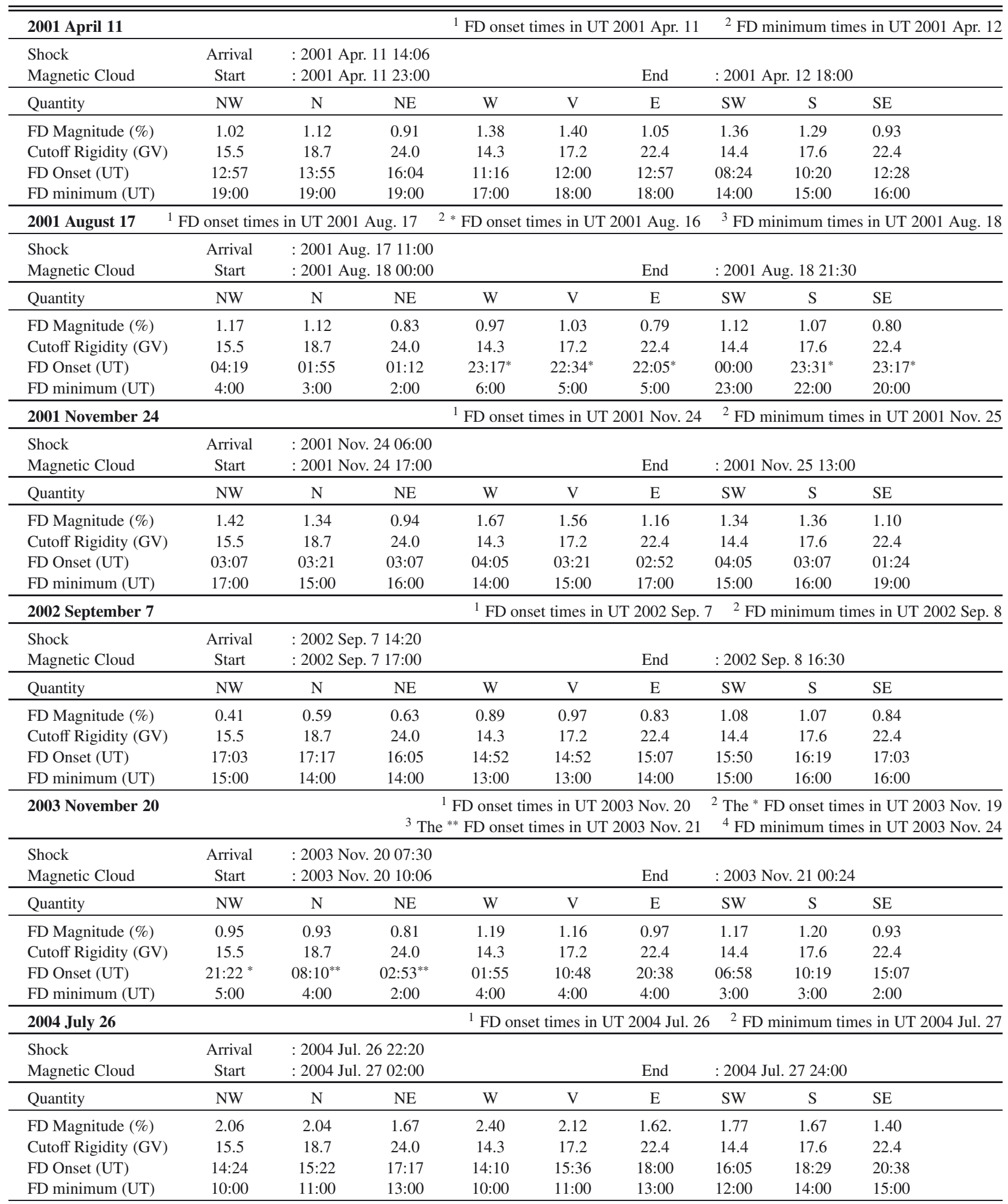

Notes. For each event, first row: magnitude of FD (FD magnitude), second row: cutoff rigidity, third row: FD onset time, fourth row: FD minimum time. The units in which each quantity is expressed is given in parentheses in the first column. 
K. P. Arunbabu et al.: High-rigidity Forbush decreases

Table A.2. Observed parameters of CME \& Shock for different events.

\begin{tabular}{|c|c|c|c|c|c|c|}
\hline Event & 2001 Apr. 11 & 2001 Aug. 17 & 2001 Nov. 24 & 2002 Sep. 7 & 2003 Nov. 20 & 2004 Jul. 26 \\
\hline \multicolumn{7}{|l|}{ CME details } \\
\hline${ }^{a}$ First obs. ( UT) & 2001 Apr. 10 05:30 & 2001 Aug. 15 23:54 & 2001 Nov. 22 22:48 & 2002 Sep. 5 16:54 & 2003 Nov. 18 8:50 & 2004 Jul. 25 14:54 \\
\hline${ }^{b} R_{\text {first }}\left(R_{\odot}\right)$ & 2.84 & 3.38 & 4.77 & 4.12 & 6.3 & 4.22 \\
\hline${ }^{c} R_{\text {last }}\left(R_{\odot}\right)$ & 18.1 & 25.9 & 25.9 & 17.0 & 27.5 & 21.9 \\
\hline${ }^{d} V_{\exp }\left(\mathrm{km} \mathrm{s}^{-1}\right)$ & 2880 & 1410 & 1370 & 1860 & 1650 & 1370 \\
\hline${ }^{e} a_{\mathrm{i}}\left(\mathrm{m} \mathrm{s}^{-2}\right)$ & 211 & -31.7 & -12.8 & 43.0 & -3.29 & 7.0 \\
\hline${ }^{f} C_{\mathrm{D}}$ & 0.325 & 0.163 & 0.09 & 0.312 & 0.333 & 0.016 \\
\hline${ }^{g} \mathrm{MC}$ start (UT) & 2001 Apr. 11 23:00 & 2001 Aug. 18 00:00 & 2001 Nov. 24 17:00 & 2002 Sep. 7 17:00 & 2003 Nov. 20 10:06 & 2004 Jul. 27 2:00 \\
\hline${ }^{h} \mathrm{MC}$ end (UT) & 2001 Apr. 12 18:00 & 2001 Aug. 18 21:30 & 2001 Nov. 25 13:00 & 2002 Sep. 8 16:30 & 2003 Nov. 21 00:24 & 2004 Jul. 27 24:00 \\
\hline${ }^{i} V_{\mathrm{sw}}^{\mathrm{MC}}\left(\mathrm{km} \mathrm{s}^{-1}\right)$ & 725 & 600 & 730 & 544 & 750 & 900 \\
\hline${ }^{j} R_{\mathrm{MC}}(\mathrm{km})$ & $2.48 \times 10^{7}$ & $2.32 \times 10^{7}$ & $2.63 \times 10^{7}$ & $2.30 \times 10^{7}$ & $1.89 \times 10^{7}$ & $3.56 \times 10^{7}$ \\
\hline${ }^{k} B_{\mathrm{MC}}(\mathrm{nT})$ & 34.5 & 25.6 & 20 & 22.9 & 50 & 25.3 \\
\hline${ }^{l} T_{\text {total }}(\mathrm{h})$ & 42.1 & 44.9 & 41.1 & 48.9 & 42.1 & 35.7 \\
\hline \multicolumn{7}{|l|}{ Shock } \\
\hline Shock arrival (UT) & 2001 Apr. 11 14:06 & 2001 Aug. 17 11:00 & 2001 Nov. 24 6:00 & 2002 Sep. 7 14:20 & 2003 Nov. 20 7:30 & 2004 Jul. 26 22:20 \\
\hline${ }^{m} B^{\mathrm{a}}(\mathrm{nT})$ & 4.5 & 5 & 5 & 5.8 & 7 & 5 \\
\hline${ }^{n} B^{\text {shock }}(\mathrm{nT})$ & 32.5 & 33 & 41.5 & 23 & & 26.1 \\
\hline${ }^{o} V_{\mathrm{sw}}^{\text {shock }}\left(\mathrm{km} \mathrm{s}^{-1}\right)$ & 670 & 501 & 948 & 550 & & 893 \\
\hline
\end{tabular}

Notes. ${ }^{(a)}$ First obs.: time in UT when the CME was first observed. ${ }^{(b)} R_{\text {first }}:$ distance in $\left(R_{\odot}\right)$, where CME was first observed. ${ }^{(c)} R_{\text {last }}:$ distance in $\left(R_{\odot}\right)$, where the CME was last observed in the LASCO FOV. ${ }^{(d)} V_{\text {exp }}$ : speed of CME in $\left(\mathrm{km} \mathrm{s}^{-1}\right)$ at $R_{\text {last. }}{ }^{(e)} a_{\mathrm{i}}$ : acceleration of CME in LASCO FOV in units of $\left(\mathrm{m} \mathrm{s}^{-2}\right){ }^{(f)} C_{\mathrm{D}}$ : the constant dimensionless drag coefficient used for the velocity profile (Sect. 2.2). ${ }^{(g)}$ MC start: Magnetic cloud start time in UT. ${ }^{(h)} \mathrm{MC}$ end: Magnetic cloud end time in UT. ${ }^{(i)} V_{\mathrm{sw}}^{\mathrm{MC}}$ : solar wind speed in $\left(\mathrm{km} \mathrm{s}^{-1}\right)$ at Earth during the arrival of the magnetic cloud. ${ }^{(j)} R_{\mathrm{MC}}$ : radius of magnetic cloud in $(\mathrm{km}){ }^{\left({ }^{(k)}\right.} B_{\mathrm{MC}}$ : magnetic field inside the magnetic cloud $(\mathrm{nT}){ }^{(l)} T_{\text {total }}$ : the Sun-Earth travel time in h for the CME. ${ }^{(m)} B^{\text {a }}$ : magnetic field in the ambient solar wind (in nT). ${ }^{(n)} B^{\text {shock}}$ : magnetic field inside the shock sheath region (in nT). ${ }^{(o)} V_{\mathrm{sw}}^{\text {shock}}$ : near-Earth shock speed $\left(\mathrm{km} \mathrm{s}^{-1}\right)$. 